\title{
Improved Cooperation in Underwater Wireless Sensor Networks
}

\author{
MUHAMMAD SHAHZAIB SANA*, MUHAMMMAD YOUSAF ALI KHAN*, NASIR SALEEM*, \\ IMRANULLAH KHAN**, AND ARBAB WAHEED AHMED***
}

RECEIVED ON 29.05.2018 ACCEPTED ON 19.02.2019

\begin{abstract}
The WSNs (Wireless Sensor Networks) lead to great opportunities to explore it scientifically. In this network different numbers of SN (Sensor Nodes) are deployed in a specific area to gather information. The UWSNs (Underwater Wireless Sensor Networks) is a highly distributed network of sensor nodes deployed underwater to gather environmental information. Hence, acquirement of real-time data at enhanced data rate and to reduce power consumption is a key concern while designing routing protocol for UWSNs. In this paper, a cooperation based solution is suggested. The solution proposed here uses the DF (Decode and Forward) strategy for relying the information from the source to the destination using a relay node. The signals coming towards the destination are weighted and combined on the basis of their SNRC (Signal to Noise Ratio Combing). The simulation results verify enhancement in different factors, required for evaluation of a UWSN. After implementation of the proposed solution the stability of the network is increased which maximize the PDR (Packet Delivery Ratio). In our proposed solution the transmission is based on channel estimation, an estimate is made for higher reliable channel, which reduces retransmission of packets. Hence, sink receive the packets with lesser delay and as a result E2E (End-to-End) delay is decreased. Data is forwarded using data forwarding by neighbor nodes. It improves average energy consumption of the system. Hence the overall performance and lifetime of a UWSN is increased.
\end{abstract}

Key Words: Wireless Sensor Network, Underwater Wireless Sensor Network, Cooperation Based Underwater Wireless Sensor Network, Improved Cooperation Techniques for Underwater Wireless Sensor Networks, Decode and Forward, Signal to Noise Ratio Combining.

\section{INTRODUCTION}

$\mathrm{T}$ he WSNs are a promising and growing area for scientific research. In WSN different sensor nodes are deployed in any area to gather desired information. The $70 \%$ of the earth's crust is covered with oceans and rivers. There is a huge opportunity to examine different features of underwater environment. The UWSN is a highly distributed network of SN deployed underwater to gather information. For underwater communication. The RF communication channels are not recommended because the radio

Authors E-Mail: (shahzaib.malik@gmail.com,myousafak@gu.edu.pk,nasirsaleem@gu.edu.pk, imrankhan031@qurtuba.edu.pk, arbabwaheed@cecos.edu.pk)

* Department of Electrical Engineering, Gomal University, Dera Ismail Khan, Pakistan.

** Department of Electrical Engineering, Qurtuba University of Science \& Information Technology, Dera Ismail Khan, Pakistan.

*** Department of Electrical Engineering, Cecos University of Information Technology \& Emerging Sciences, Peshawar, Pakistan.

This is an open access article published by Mehran University Research Journal of Engineering and Technology, Jamshoro under the CC by 4.0 International License. 
frequency spectrum has limited wave propagation and faces larger noise in underwater channel. The Acoustic signals are used as a communication channel for underwater communication. It is preferred because it is more robust and reliable, but it has limited bandwidth. In UWSNs the SN are supplied with a limited amount of power backup, it is very important for UWSNs to manage their power expenditure effectively to enhance network lifetime. To obtain real-time data at enhanced data rate and to reduce power consumption of the $\mathrm{SN}$, it is always kept in mind while designing UWSNs.

Davs and Chang [1] discussed different applications and challenges to UWSNs. Das et. al. [2] discussed a comparison between WSN and UWSNs in terms of energy consumption, medium characteristics, routing and topology challenges. Rao et. al. [3] discussed different architecture schemes like static, semi-mobile, mobile architectures, single hop, multi-hop and hybrid architectures. They have been proposed an AM-DisCNT Angular Multi-Hop Distance based Circular Network Transmission Protocol. This technique is fabricated by different $\mathrm{SN}$ that forms clusters in a particular area, the $\mathrm{SN}$ with larger amount of energy serve as $\mathrm{CH}$ (Cluster Head) for a specific round of transmission of data to the sink node. Khalid et. al. [4] categorized different protocols on the bases of localization. Ahsan et. al. [5] has discussed different types of protocols for UWSNs, they grouped them into three categories i.e. Clustering-based routing, Localization-free routing and Cooperation-based routing. Different types of protocols of said categories have also discussed. Jafri et. al. [6] suggested an AMCTD (Adaptive Mobility of Courier Nodes in Threshold-Optimized DepthBased) routing protocol. In AMCTD, the network lifetime was enhanced by implementation of optimal weight functions. This proposal consists of 3 stages; weight updating, depth threshold variation and adaptive mobility of courier nodes.

Sheeraz et. al. [7] and Li et. al. [8] proposed Co-UWSN (Cooperative UWSN). In these solutions relays are nominated on the basis of their distance and SNR calculations of the channel conditions. Sheeraz et. al. [9] proposed ARCUN (Analytical Approach towards Reliability with Cooperation for UWSNs). Kumar et. al. [10] suggested OVAR (Opportunistic Void Avoidance Routing) protocol. Heidemann et. al. [11] have designed EEDBR (Energy-Efficient Depth-Based Routing) protocol. Benslimane et. al.[12] categorized different relaying schemes for cooperative communication. Georgy et. al. [13] discussed AF and DF relying strategies. Nigatu, and Lawrence [14] discussed different combining strategies for destination nodes.

\section{COOPERATION BASED MODELING}

We consider a situation of WSN that contains "Ni" SNs randomly deployed in a particular area underwater. In this model every SN has two roles, either it works as "source" to send its data or to work as "relay" to assist other nodes by sending their data to the destination. Let " $\mathrm{S}_{\mathrm{i}}$ " is ith number of source nodes and " $\mathrm{R}_{\mathrm{i}}$ " is ith number of relay nodes. Let " $\mathrm{A}$ " be the area under sea where these SNs are randomly deployed. Consider " $L$ " be the total number of links creating a path between different sensor nodes. Every sensor node contains single omnidirectional antenna that is considered to have adjustable transmission power. Initially all sensor nodes are considered to have identical maximum power of transmission $\mathrm{P}_{\max }$. The range for communication of each $\mathrm{SN}$ is directly proportional to the $\mathrm{P}_{\max }$. 


\subsection{Cooperation Model}

This transmission mechanism ensures a distinct transmission between source node and relay. Fig. 1 represents a 3 nodes cooperation model. we use multi hop mechanism for transmission of data, each source node uses less power to transfer its data towards it neighbor. Through this data forwarding technique using neighbors, the data is send towards destination by consuming less power as that of an individual node respectively. This mechanism works in two-steps.

In the $1^{\text {st }}$ step the $S$ (Source) directs data towards the D (Destination) and the R (Relay) simultaneously, and in the $2^{\text {nd }}$ step " $R$ " sends the received data to " $D$ ". Total distance between source and destination is " $\mathrm{d}_{1}+$ $\mathrm{d}_{2}$ "where, " $\mathrm{d}_{1}$ " is separation between source and relay pair while the relay and destination are separated by the distance " $\mathrm{d}_{2}$ ".

The mathematical model for first step of transmission is given in Equation (1-2):

$\mathrm{Y}_{\mathrm{SR}}=\mathrm{h}_{\mathrm{SR}} \cdot \mathrm{x}_{\mathrm{S}}+\eta_{\mathrm{SR}}$

$\mathrm{Y}_{\mathrm{SD}}=\mathrm{h}_{\mathrm{SD}} \cdot \mathrm{x}_{\mathrm{S}}+\eta_{\mathrm{SD}}$

Here the $\mathrm{Y}_{\mathrm{SR}}$ is the data symbol received from the source to relay and $\mathrm{Y}_{\mathrm{SD}}$ is the data symbol received from source to destination node respectively. The $\mathrm{X}_{\mathrm{S}}$ is the forwarded symbol, $\eta_{\mathrm{SR}}$ and $\eta_{\mathrm{SD}}$ are the channel noises from the source to the relay and from the relay to the destination respectively. The $h_{S R}$ and $h_{S D}$ are path coefficients from the source to the relay and the source to the destination node correspondingly. The $\mathrm{h}_{\mathrm{SR}}$ and $\mathrm{h}_{\mathrm{SD}}$ are stated in Gaussian random variable $\mathrm{CN}(0, \sigma 2)$ where " $\sigma 2$ " is the variance and " 0 " is its arithmetic mean.

In the second step, the relay implements DF processing technique on received data and then re-transmits it to the destination. The mathematical equation of the second step is given in Equation (3):

$\mathrm{y}_{\mathrm{RD}}=\mathrm{h}_{\mathrm{RD}} \mathrm{x}_{\mathrm{S}} \mathrm{f}_{(\mathrm{ySR})}+\mathrm{h}_{\mathrm{RD}}$

The $\mathrm{f}_{(\mathrm{ySR})}$ represents the processing function on the received symbol. The $\eta_{R D}$ is the noise of channel between relay and destination.

\subsection{Network Model}

We consider a "K-hop" cooperative channel which is designed by using communication techniques. In this model every SN has two roles; either it works as "source" to send its data or work as "relay" to assist other node by transmission of their data. Hence, this transmission mechanism allows a non-overlapping transmission of source node and relay. The bandwidth of around 10-32

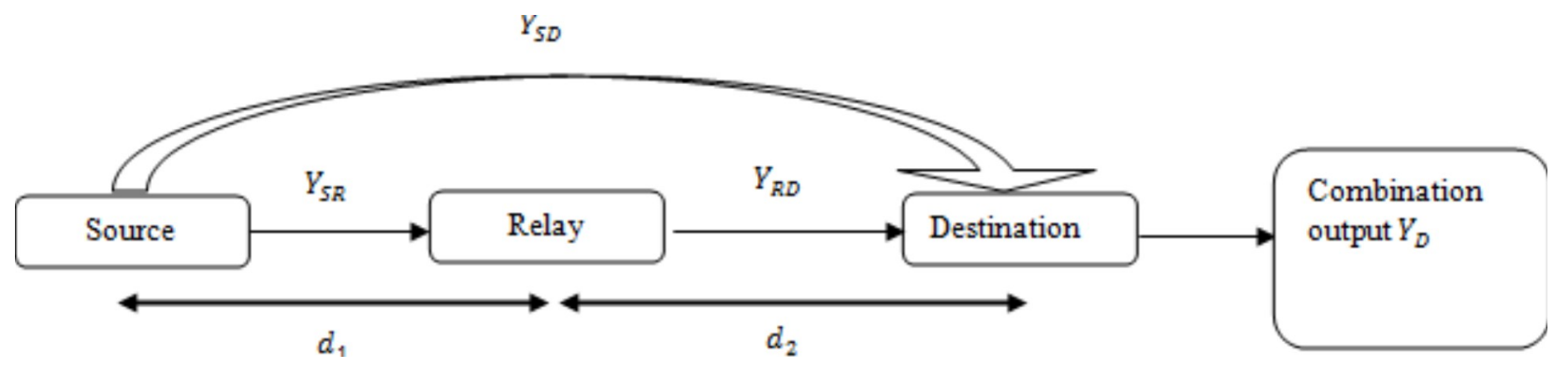

FIG. 1. LINEAR 3 NODES MODEL 
$\mathrm{kHz}$ is used for underwater communication. The multiband communication signals divide the entire frequency band (10-32 kHz) into four sub-bands each with bandwidth of $4.5 \mathrm{kHz}$, centered at $12,18,24$, and $30 \mathrm{kHz}$, respectively. The probability of overlapping is low and because of that reason non-overlapping channel have been assumed [15]. For a group of receivers $r_{i} \varepsilon R_{k}$ and transmitters $t \varepsilon T_{k}$ the channel is in series with l-number of links $\left(1_{1}, \ldots \ldots \ldots 1_{\mathrm{i}}\right)$ ) that are cooperative. It links the $i$-sources to the $r$ destinations in a non-overlapping channel. The main objective is to reduce E2E energy consumption for successful communication towards sea sink.

We consider the cost of link $1_{\mathrm{i}}$ is $C\left(\mathrm{~T}_{\mathrm{K}}, \mathrm{R}_{\mathrm{K}}\right)=\mathrm{P}_{\mathrm{li}}$. It is lowest power required to generate cooperation based edge in single loop cooperative communication. The main objective is to find shortest distance between source and the destination to minimize net power of transmission. It will obtain the desire throughput $(\eta)$ and the transmission will face smallest amount of $\operatorname{SNR}(\gamma)$. For a given link $1_{i}$,

$\operatorname{Minimize}\left(\sum_{1_{i} \varepsilon L} P_{l i}\right)$

With subject to

$$
\left\{\begin{array}{l}
\sum_{i=1}^{\mathrm{L}} \eta_{\mathrm{l}_{i}} \geq \eta_{\mathrm{o}} \\
\text { and } \\
\sum_{\mathrm{i}=1}^{\mathrm{L}} \gamma_{\mathrm{l}_{\mathrm{i}}} \geq \gamma_{\mathrm{o}}
\end{array}\right.
$$

Here the $\eta_{\mathrm{li}}$ is throughput of ith link, end to end throughput is represented by $\eta_{0}$, while probabilistic SNR is represented by $\gamma_{0}$ and $\gamma_{\mathrm{li}}$ is SNR of ith link which is constant throughout the link. For transmitters $\mathrm{k} \mathrm{R}_{\mathrm{i}}$ or $\mathrm{k} \varepsilon\left\{0,1,2,3,{ }_{,}, \mathrm{R}_{\mathrm{i}}\right\}$ the powers of cooperative transmissions is $\mathrm{P}_{\mathrm{Rk}}$.

The Equation (4) may be stated as:

$\min \sum_{\mathrm{l}_{\mathrm{i}} \varepsilon \mathrm{L}} \mathrm{P}_{\text {co }}-\mathrm{l}_{\mathrm{i}}=\operatorname{minimize} \sum_{\mathrm{l}_{\mathrm{i}} \varepsilon \mathrm{L}}\left\lfloor\mathrm{P}_{\mathrm{b}, \mathrm{i}}+\sum_{\mathrm{k}=0}^{\mathrm{R}} \mathrm{P}_{\mathrm{R}_{\mathrm{k}}}\right\rfloor$
With subject to,

$$
\left\{\begin{array}{l}
\sum_{i=1}^{L} \eta_{l i} \geq \eta_{o} \\
\text { and } \\
\sum_{i=1}^{L} \gamma_{1} \geq \gamma_{o}
\end{array}\right.
$$

Where $\mathrm{P}_{c o}-1_{\mathrm{i}}$, is the power of cooperative communication for a link $1_{i}$ and node $i$.

\subsection{Channel Model}

We assume a " $\mathrm{t}_{\mathrm{i}}$ " node, forwarding its data " $\mathrm{x}_{\mathrm{i}}$ " to the receiving node " $r_{i}$ " which is accepting data " $y_{j}$ " in direct phase or broadcasting. The " $\mathrm{x}_{\mathrm{i}}$ " has the unity power while " $\mathrm{t}_{\mathrm{i}}$ " transmitter has the ability to withstand its power $\mathrm{P}_{\mathrm{b}, \mathrm{i}}$ to $\mathrm{P}_{\max }$. Data received at $\mathrm{r}_{\mathrm{j}}$ can be denoted by Equation (6).

$y_{j}^{d}=\sqrt{\frac{p_{b, i}^{d}}{p_{i j}^{a}}} h_{i j} \cdot x_{i}+N_{j}$

Where, the $\mathrm{N}_{\mathrm{j}}$ is the noise of underwater channel, and the $r_{j}$ represent interference in the UW channel. The " $d$ " represents the direct transmission at superscript. Where the " $\mathrm{d}_{\mathrm{ij}}$ ", is separation between " $\mathrm{r}_{\mathrm{j}}$ " and the node " $\mathrm{t}_{\mathrm{i}}$ ". The " $\alpha$ " is path loss and it normally varies from $1-3$. The $\mathrm{h}_{\mathrm{ij}}$ is the complex channel gain among " $\mathrm{t}_{\mathrm{i}}$ " and " $\mathrm{r}_{\mathrm{j}}$ ", it can also be formulated as Equation (7).

$h_{i j}=\left|h_{i j}\right| e^{j \theta_{i J}}$

Here, the $\theta_{\mathrm{ij}}$ is the phase of channel, $\left|h_{i j}\right|$ is the magnitude of the channel gain, it has the Rayleigh Distribution having a unit power of $E\left[\left.h_{i j}\right|^{2}\right]=1$ it also expressed as 
$h_{i j} \approx C N\left(0, \sigma_{i j}^{2}\right)$ with $\sigma_{\mathrm{ij}}^{2}=E\left[\left|h_{i j}^{2}\right|\right]=1$

At $r_{j}$ the power receiving is

$$
P_{j}^{d}=\sum_{t_{i} \varepsilon T}\left(\left|h_{i j}\right|^{2} / d_{i j}^{a}\right) P_{b, i}^{d}
$$

Here $\mathrm{T}$ demonstrating a combination of all nodes sending their data. By considering $\gamma^{\mathrm{d}}{ }_{\mathrm{ij}}$ is the SNR at $\mathrm{r}_{\mathrm{j}}$ receiver then,

$\gamma_{i j}^{d}=\bar{\gamma}\left|h_{i j}^{2}\right|=\frac{1}{d_{i j}^{a}} \frac{p_{b, i}^{a}}{p_{n_{n}}} \cdot\left|h_{i j}\right|^{2}$

Here $\mathrm{P}_{\mathrm{nj}}$ is the noise power at the receiver " $\mathrm{r}_{\mathrm{j}} \cdot \mathrm{As}\left|\mathrm{h}_{\mathrm{ij}}\right|^{2}=1$, so Equation (9) can be written as:

$\gamma_{i j}^{d}=\bar{\gamma}\left|h_{i j}^{2}\right|=\frac{1}{d_{i j}^{a}} \frac{p_{b, i}^{a}}{p_{n_{j}}}$

where

$\bar{\gamma}=\operatorname{AverageSNR}(\mathrm{d}, \mathrm{f})=\frac{\mathrm{P}_{\mathrm{xj}} / \mathrm{A}(\mathrm{d}, \mathrm{f})}{\mathrm{N}_{\mathrm{f}}(\mathrm{f}) \mathrm{B}}$

Here the $B$ is used for the bandwidth for the $x_{j}$ transmitted signal having the power $\mathrm{P}_{\mathrm{xj}}$.

\subsection{Channel's Reliability}

The technological advancement and inventions of many useful techniques has decrease the data loss due to outage of the channel. We also focused on link reliability to achieve diversity. The results of different combining strategies may apply to achieve diversity and reliability.
Many links join together to make hops, then these channels make a sequence of nodes that communicate with each other, the whole process complete a channel between the $\mathrm{S}$ and $\mathrm{D}$. The transmission will consider as successful when entire data is transferred from the source to the destination. The probability of end to end reliability can be represented as "!, it may be formulated as Equation $(12-13)$

$\mathfrak{R}=1-P_{\text {outage }}$

$\mathfrak{R}=1\left\{2 \pi H \times \exp \left(\frac{2^{R / B}+S L+\rho(d, f)}{20}\right)\right\}$

Where $\mathfrak{R}$ is representing a function that established on separation between two nodes, their depth in water and channel's state.

From Equation (13), the total reliability can be calculated for a complete end to end channel:

$$
\mathfrak{R}=1-\left[\sum_{i=1}^{n}\left\{2 \pi H_{i} \times \exp \left(\frac{2^{R / B}+S L+\rho(d, f)}{20}\right)\right\}\right]
$$

For the path that has reduced distance or minimum distance and decrease this sum will have maximum reliability.

\subsection{Relay Strategy}

In the existing scheme [5] the authors used AF (Amplify and Forward) technique at relay, which is more energy consuming. Here we used DF (Decode and Forward) technique as relay strategy. The Equation (15) is represent DF Equation (16),

$\mathrm{Y}_{1}=\mathrm{h}_{1} \mathrm{x}_{0}+\varepsilon_{1}, \mathrm{Y}_{2}=\mathrm{h}_{2} \mathrm{x}_{1}+\varepsilon_{2}$ 
By combining the values of Equation (16)

$\mathrm{Y}=\mathrm{h}_{\mathrm{N}} \mathrm{x}_{\mathrm{N}-1}+\varepsilon_{\mathrm{N}}$

Here, $\mathrm{x}_{\mathrm{a}}, \mathrm{y}_{\mathrm{a}}$ and $\varepsilon_{\mathrm{a}}($ for $\mathrm{a}=1,2,3 \ldots \mathrm{N}-1)$ are the signs being transmitted and received at relay.

\subsection{Combining Strategy}

The combining strategies used to combine a signal that is coming from multiple sources. As the destination node received packets from multiple paths, so these packets have to recombine at the destination. We used Signal to SNRC for the signals coming from the source and the relays.

The SNR is parameter that is used to analyze the quality of the channel. This can also be utilized to weight the received signals. This is represented by Equations (17-18).

$y_{D}[n]=\sum_{i=1}^{k} S N R_{i} \times y_{i, d}[n]$

here $y_{d}[n]$ denotes the total signal at the receiver and $\mathrm{y}_{\text {id }}[\mathrm{n}]$ is the signal from the ith link.

For a single relay,

$\mathrm{y}_{\mathrm{D}}[\mathrm{n}]=\mathrm{SNR}_{\mathrm{d}} \mathrm{xy}_{\mathrm{d}}[\mathrm{n}]+\operatorname{SNR}_{\mathrm{r}} \mathrm{xy}_{\mathrm{r}}[\mathrm{n}]$

Here $\mathrm{SNR}_{\mathrm{d}}$ is SNR of direct link, whereas $\mathrm{SNR}_{\mathrm{r}}$ is SNR of link over relay.

\section{SIMULATION RESULTS}

The MATLAB simulation has been done to analyze the performance of the IM-COUWSN. For this purpose, we simulate an underwater environment that covers an area of 500 square meters. The $\mathrm{BW}$ is around $10 \mathrm{~Kb}$ on each medium. We deployed 225 nodes that are followed by 10 sinks on surface. There can be three or more than three nodes between source node and sink nodes, however the delay is around 0.05 seconds. This pattern has been randomly varied for 5000 rounds of simulation by considering multiple sink models. We assume 100 meters as range of transmission for sensor node that also consider different physical features of underwater acoustic model. After every successful communication all active nodes transfer threshold based data to sink. After every round, all active nodes transfer their threshold-based data to the sink. Every node transfers essential physical parameters, especially depth threshold and weight with its neighbors to update them with the varying conditions of the UWSN. After every 100th round, the alive nodes calculate their distance from the neighbor nodes. The nodes transmit their data to the higher layer using forwarding nodes, till the data is received at desired destination. The sink manages the depth of thresholds and the adaptive mobility of cooperating nodes. It is quite expensive but also beneficial as it improves overall performance of the network.

\subsection{Network Lifetime v/s Stability Period}

It is the measure of time between initialization of the network to the time where $1^{\text {st }} \mathrm{SN}$ of network stops transmission of sensed data. This time is termed as stable period of the network. Whereas, unstable period of network start after the death of $1^{\text {st }}$ node, persist to the expiry of last alive SN. Higher value of stability period ensures better output in terms of stability.

Mehran University Research Journal of Engineering \& Technology, Volume 38, No. 4, October, 2019 [p-ISSN: 0254-7821, e-ISSN: 2413-7219] 
It is an important KPI (Key Performance Indicator) for UWSNs. The IM-COUWSN improves the network's stability period by ensuring maximum number of alive nodes. It is done by keeping less transmission losses and by reducing transmission of redundant data. In simulation of CO-UWSN the first node dies at $980^{\text {th }}$ round of total 10,000 rounds [5], but in the IM-COUWSN it works effectively till $3200^{\text {th }}$ round. Thus it improves the stability of network which as shown in Fig. 2.

After $3200^{\text {th }}$ round the stability of network start declining gradually. It is happened when initial nodes become died, hence network disrupts because of lack of alive neighbors. The stability time of the IM-COUWSN is higher than the CO-UWSN [5] because of effective management of energy consumption. In CO-UWSN, total numbers of neighbor decreases as network become sparse that cause stability issues. CO-UWSN considers depth and residual energy as forwarding elements which produces trade-off between lifetime and transmission losses of network. Moreover, in CO-UWSN as network become sparse it produces load on nodes having higher energy [5]. The lifetime of the IM-COUWSN can be enhanced by lowering throughput of sensors network. We employed Thorps Energy Model which postulates the complete channel losses and it is valuable for discriminating data forwarding in responsive networks.

\subsection{Network Lifetime v/s End-to-End delay}

It is the time required for a packet to travel through the network from its source towards destination. It is another KPI for UWSNs. The IM-COUWS improves the E2E delay which is achieved by minimizing the forwarding distance between SN. The Fig. 3 shows the result of three simulation rounds.

For the CO-UWSN this E2E delay is higher for startup conditions because of larger the distance between SNs. The E2E delay than decreases as the system complete initial 500 rounds, because at that time network become sparse.

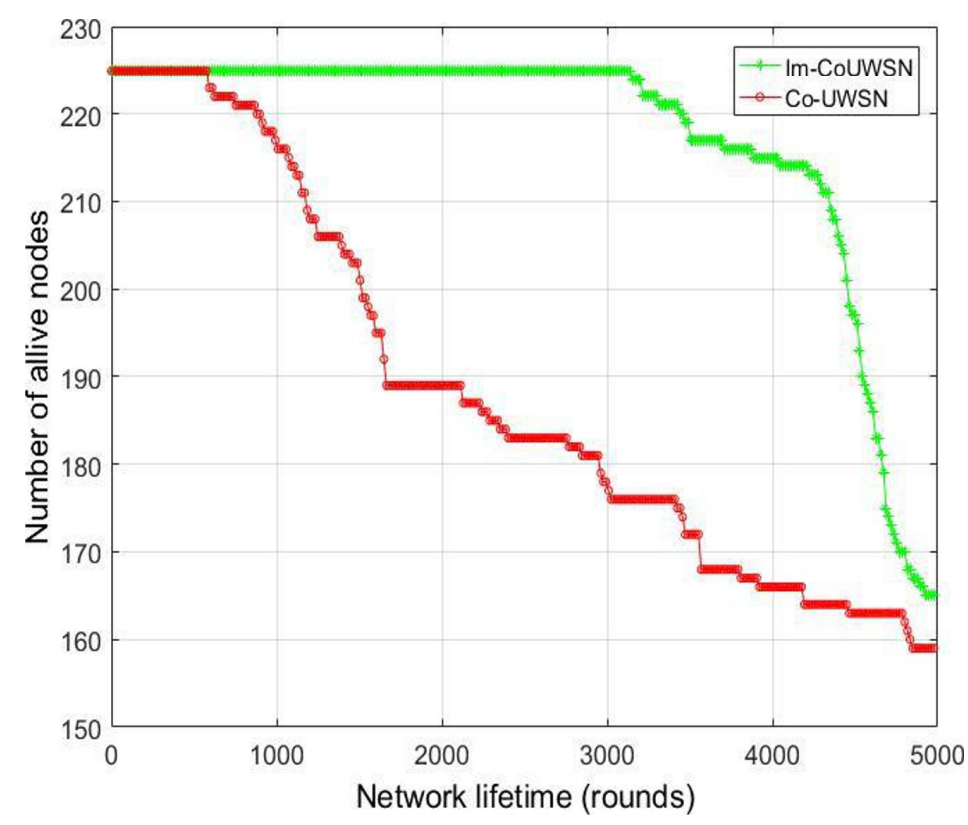

FIG. 2. NETWORK LIFETIME V/S STABILITY PERIOD 
In the IM-COUWSN the E2E delay is better than the COUWSN, because of load balancing.

The CO-UWSN transfer packets with least number of hops but due to low quality channel, the packet loss increases at the destination. Hence, the packets need to be transmitted again. This retransmission of lost packet also causes E2E packet delay [5]. In IM-COUWSN transmission is based on channel estimation, an estimate is made for higher reliable channel, which reduce retransmission of packets. Because of this estimation in the IM-COUWSN the sink received packet with lesser delay.

\subsection{Network Lifetime v/s Energy Consumption}

It is the energy consumed by network from the initialization state to the state where last SN dies. This represents total energy consumed by network for its operations. The total energy consumption is always a key concern while designing routing protocols for the UWSNs. In the IM-COUWSN, we introduced data forwarding using neighbor nodes that achieve load balancing and hence it improved average energy consumption. The implementation of proper weight functions also improving average energy consumption. By effectively utilizing forwarding nodes and the depth difference, the IM-COUWSN is a better choice for timecritical applications of UWSNs. In the IM-COUWSN the nodes may consume larger amount of energy while transmitting with distant nodes, however it is lesser then energy consumption in CO-UWSN technique where high energy nodes are repeatedly selected for transmission.

The Fig. 4 represents the comparison of total energy consumption of Co-UWSN and IM-COWSN.

\subsection{Network Lifetime v/s Path Losses}

The path losses are the loss of channel through which a packet is travelling it is also known as path attenuation. In the CO-UWSN, the transmission loss

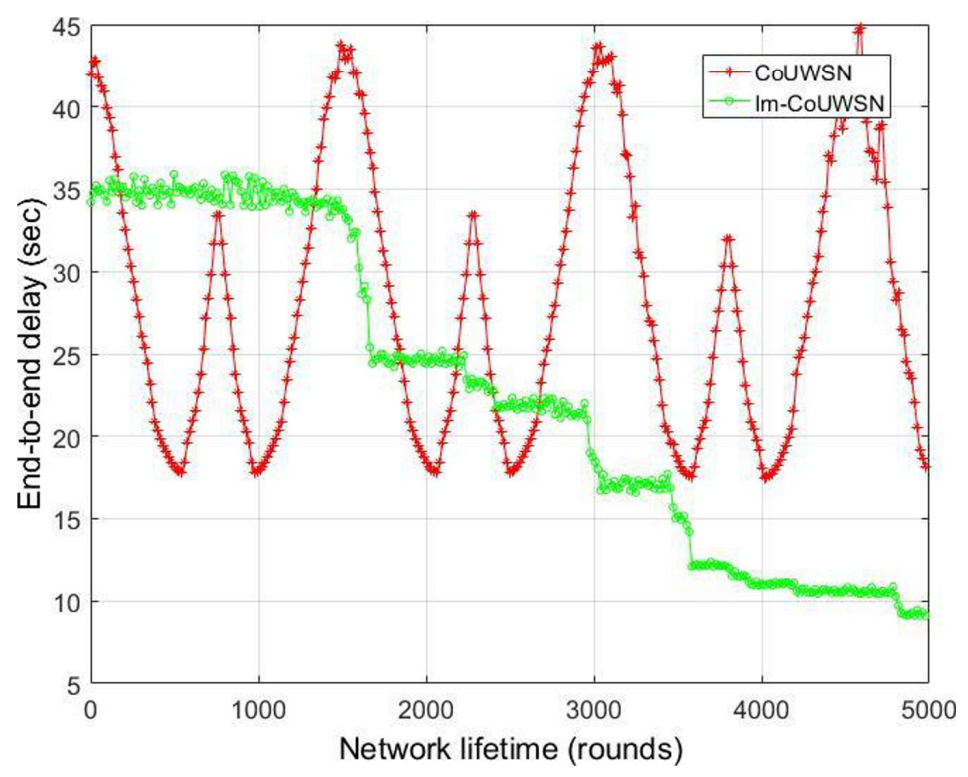

FIG. 3. NETWORK LIFETIME V/S END-TO-END DELAY

Mehran University Research Journal of Engineering \& Technology, Volume 38, No. 4, October, 2019 [p-ISSN: 0254-7821, e-ISSN: 2413-7219] 1016 
is increased for multiple transmissions between source and destination, but in IM-COUWSN, there are preferences for distant transmissions hence it decreases transmission loss. The Fig. 5 is representing a comparison between Network Lifetime and Path
Losses. This is achieved by deploying Thorps Attenuation Model to calculate path losses. We also used the Urick's Model to calculate effects of pathloss on frequency of transmission, bandwidth efficiency, and noise density during transmission.

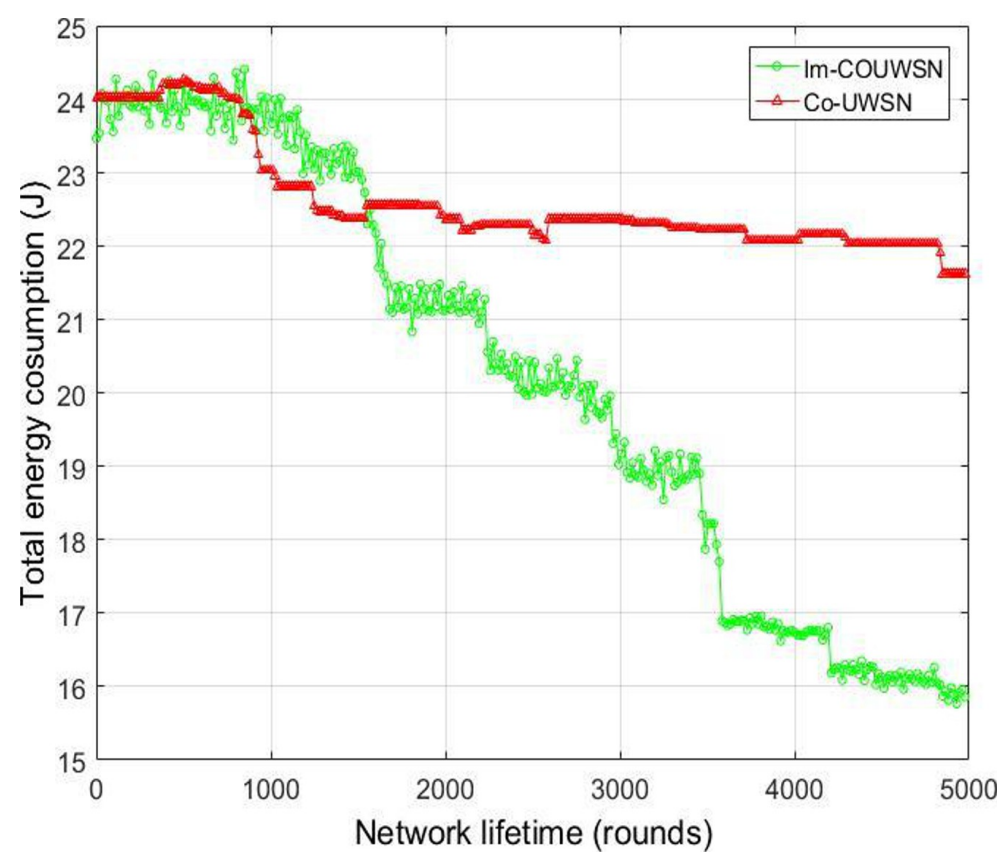

FIG. 4. NETWORK LIFETIME V/S ENERGY CONSUMPTION

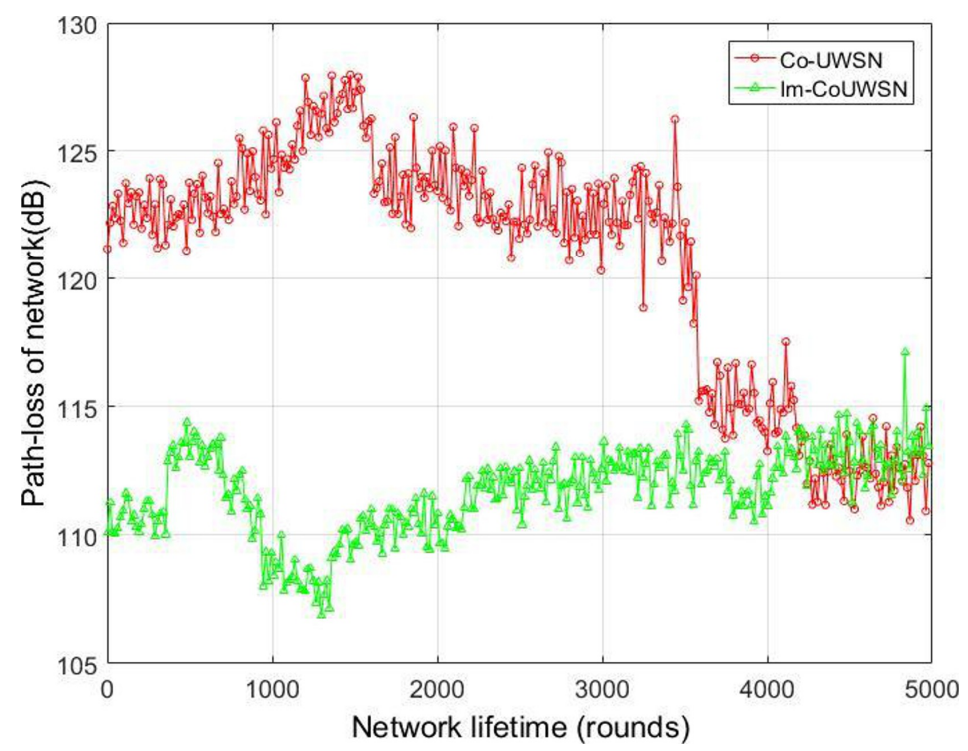

FIG. 5. NETWORK LIFETIME V/S PATH-LOSS

Mehran University Research Journal of Engineering \& Technology, Volume 38, No. 4, October, 2019 [p-ISSN: 0254-7821, e-ISSN: 2413-7219] 1017 
During startup condition of the network, the CO-UWSN confirm lesser losses because of higher network density. When the network become sparse, the packet loss increases which abruptly decreases the performance of UWSN. It happens as their is non-availability of alive neighbors to transfer packets. These losses are significantly decreased in the IM-COUWSN as SNRC keep in account of both residual energy and depth of relay. The introduction of forwarding nodes in the IMCOUWSN also improved path losses. After $3500^{\text {th }}$ round of simulation, packet loss gradually increases because of shortage of relay nodes.

\subsection{Network Lifetime v/s Packet Delivery Ratio}

It is a fraction of the number of packets received to the number of packets transmitted. It is an important parameter to examine the performance of any routing protocol designed for UWSN. In Fig. 6, a relationship has shown between the IM-COUWSN and the COUWSN.

It shows that the PDR is comparatively better in the IMCOUWSN than the CO-UWSN. The higher traffic is observed between nodes for lesser inter-arrival time for packets; it increases the packet collision and decreases the PDR. The IM-COUWSN increases the probability of PDR by forwarding packets on multiple links. In the COUWSN multiple forwarding is employed for distant propagations hence it decreases PDR.

In the IM-COUWSN we consider depth and residual energy functions for data forwarding which results a stable propagation. After $3000^{\text {th }}$ round of simulation, there is a drop in PDR which results in packet loss and delay. The higher reliability can be achieved by higher number of cooperating nodes.

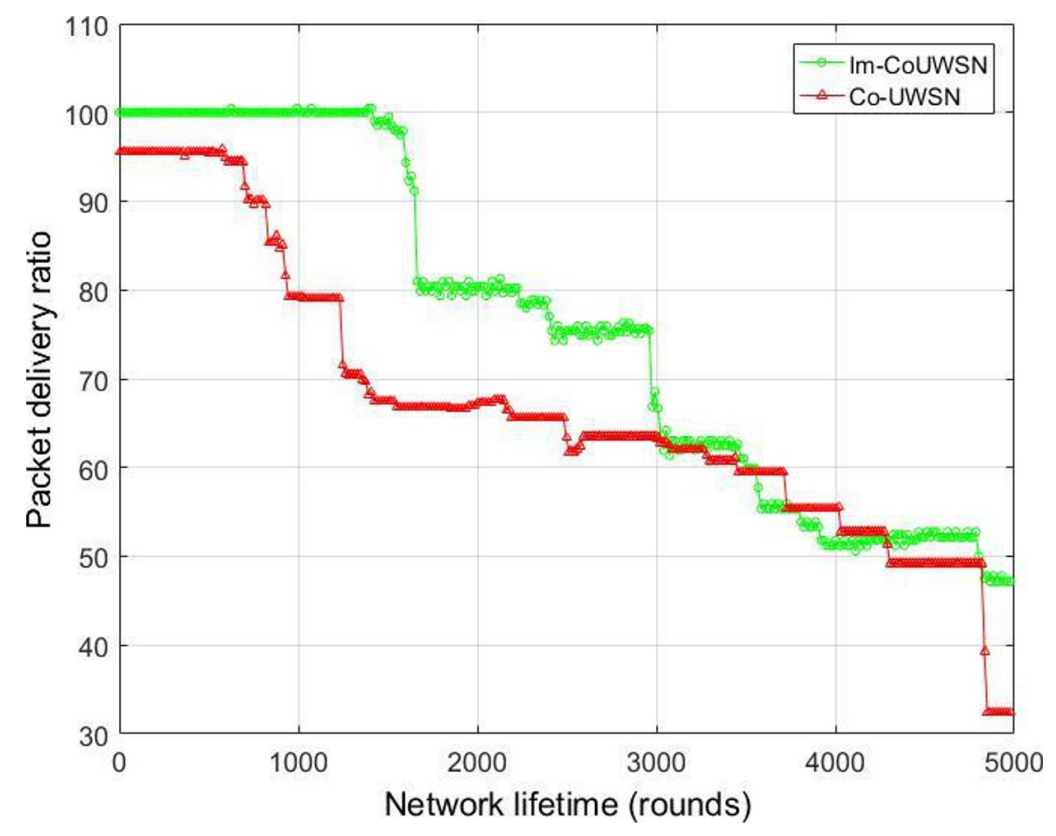

FIG. 6. NETWORK LIFETIME V/S PACKET DELIVERY RATIO

Mehran University Research Journal of Engineering \& Technology, Volume 38, No. 4, October, 2019 [p-ISSN: 0254-7821, e-ISSN: 2413-7219] 


\section{CONCLUSION}

This paper has recommended a new routing scheme based on cooperation techniques. This proposed solution has Decode and Forward strategy for relying the information from the source node to the destination node. The incoming signals are weighted and combined on the basis of their Signal to SNRC. The simulation results also verified enhancement in different factors, required for evaluation the UWSN. After the implementation of our proposed solution the stability of the network increases. It maximizes the PDR. It also decreases the E2E delay. The introduction of data forwarding using neighbor nodes achieved load balancing and hence, improved average energy consumption effectively. Thus, overall performance and the lifetime of a UWSN have increased. This also verifies that our proposed scheme has improves the results of existing routing techniques based on cooperation's for UWSNs.

\section{ACKNOWLEDGEMENTS}

This research work is supported by Faculty of Engineering \& Technology, Gomal University, Dera Ismail Khan, Pakistan. The authors are thankful to all those members who provided the required resources to perform this research.

\section{REFRENCES}

[1] Almir, D., and Chang, H., "Underwater Wireless Sensor Networks", IEEE Conference on Underwater Wireless Sensor Networks, Oceans 2012.

[2] Aiswarya, D., Kumar, K.V., and Rao. M., "Current State of Art Techniques in Underwater Wireless Sensor Network", International Journal of Emerging Trends \& Technology in Computer Science, Volume 6, No. 3, pp. 273-281, 2017.

[3] Rao, A., Akbar, M., Javaid, N., Saad, N.M., and Sarfraz, S., "AM-DisCNT: Angular Multi-Hop Distance Based Circular Network Transmission Protocol for WSNs", IEEE $8^{\text {th }}$ International Conference on Broadband and Wireless Computing, Communication and Applications, pp. 29-35, 2013.
[4] Khalid, M., Ullah, Z., Ahmad, N., Arshad, M., Jan, B., Cao, Y., and Adnan, A., "A Survey of Routing Issues and Associated Protocols in Underwater Wireless Sensor Networks", Journal of Sensors, pp 1-17, 2017.

[5] Muhammad, A., Ahmed, S., Hadi, F., Wahab, F., and Ahmed, I., "A Recent Study on Routing Protocols in UWSNs", International Journal of Advanced Computer Science and Applications, Volume 8, No. 4, pp. 431-435, 2017.

[6] Jafri, M.R., Ahmed, S., Javaid, N., Ahmad, Z., and Qureshi, R.J., "AMCTD: Adaptive Mobility of Courier Nodes in Threshold-Optimized DBR Protocol for Underwater Wireless Sensor Networks", IEEE International Conference on Broadband and Wireless Computing, Communication and Applications, pp. 93-99, 2013.

[7] Ahmed S., Javaid, N., Khan, F.A., Durrani, M.Y., Ali, A., Shaukat, A., Sandhu, M.M., Khan, Z.A., and Qasim, U., "Co-UWSN: Cooperative Energy-Efficient Protocol for Underwater WSNs", International Journal of Distributed Sensor Networks, 2015.

[8] Li, Z., Yao, N., and Gao, Q., "RDBF: Relative DistanceBased Forwarding Protocol for Underwater Wireless Networks", International Journal of Distributed Sensor Networks, pp 1-11, 2014.

[9] Ahmed, S., Akbar, M., Ullah, R., Ahmed, S., Raza, M., Khan, Z.A., Qasim, U., and Javaid, N., "ARCUN: Analytical Approach Towards Reliability with Cooperation for Underwater WSNs", Procedia Computer Science, Volume 52, pp. 576-583, 2015.

[10] Kumar, V., Naresh, M., Kumar, S., Rajakumari, J., and Mohanarangan, S., "Opportunistic Void Avoidance Routing for Underwater Sensor Networks", International Journal of Scientific Research in Computer Science, Engineering and Information Technology, Volume 2, pp. 924-930, 2017.

[11] Heidemann, J., Ye, W., Wills, J., Syed, A., and Li,Y.,"Research Challenges and Applications for Underwater Sensor Networking", IEEE Conference on Wireless Communications and Networking, pp. 228-235, Las Vegas, USA, April, 2006. 
[12] Benslimane, M., Ibriz, A., and Mostafa, H., "Cooperative Communication and Cooperation Techniques in Sensor Networks", Journal of Theoretical \& Applied Information Technology, Volume 57, No. 1, 2013.

[13] Georgy, L., and loyka, S., "Amplify-and-Forward Versus Decode-and-Forward Relaying: Which is Better?”, 22th International Zurich Seminar on Communications, pp. 123-126, Zurich, Switzerland, March, 2012
[14]

Nigatu, M.D., and Lawrence, O.C., "Cooperative Communication Techniques in Wireless Networks (Analysis with Variable Relay Positioning)", Master's Thesis, Blekinge Institute of Technology School of Engineering, 2010.

[15] Song, H.C., and Hodgkiss, W.S., "Efficient Use of Bandwidth for Underwater Acoustic Communication", The Journal of the Acoustical Society of America, Volume 134, No. 2, pp. 905-908. 2013. 